\title{
AVALIAÇÃO TÉCNICA E DE CUSTOS DO TRANSPORTE RODOVIÁRIO COM DIFERENTES ESPÉCIES E SORTIMENTOS DE MADEIRA
}

\author{
Eduardo da Silva Lopes ${ }^{1 *}$, Thábata Palma Vieira ${ }^{2}$, Carla Krulikowski Rodrigues ${ }^{3}$ \\ ${ }^{1}$ Universidade Estadual do Centro-Oeste, Departamento de Engenharia Florestal, Irati, Paraná, Brasil - eslopes@pq.cnpq.br ${ }^{*}$ \\ ${ }^{2}$ Universidade Estadual do Centro-Oeste, Curso de Engenharia Florestal, Irati, Paraná, Brasil - thabata.vieira@ @otmail.com \\ ${ }^{3}$ Universidade Estadual do Centro-Oeste, Programa de Pós-Graduação em Ciências Florestais, Irati, Paraná, Brasil - carlakr@ gmail.com
}

Recebido para publicação: 14/06/2014 - Aceito para publicação: 20/05/2016

\begin{abstract}
Resumo
Este trabalho objetivou realizar uma avaliação técnica e de custos do transporte florestal rodoviário com diferentes espécies e sortimentos de madeira. $\mathrm{O}$ estudo foi realizado com dados obtidos em uma empresa transportadora localizada no estado do Paraná. Foi analisado o ciclo operacional do transporte de madeira, determinando-se a disponibilidade mecânica, a eficiência operacional, a produtividade e os custos do veículo "bitrem" transportando toras de Eucalyptus sp. e Pinus sp. com 3,60 e 7,20 m de comprimento. Os resultados mostraram que os tempos consumidos pelos veículos nas filas de espera para o carregamento e descarregamento influenciou na redução da produtividade e no aumento dos custos do transporte. $\mathrm{O}$ comprimento da madeira é uma variável que afetou diretamente as operações de carregamento e descarregamento.

Palavras-chave: Produtividade; custo; logística; otimização.
\end{abstract}

\begin{abstract}
Technical and cost evaluation of road transportation with different species and assortments wood. The objective of this study was to realize a technical and cost evaluate of the truck forest transportation with different species and wood assortments. The study was conducted with data of a trucking company located in the Paraná State. The operational cycle of the wood transportation was analyzed, determining the mechanical availability, operational efficiency, productivity and costs of "bitrem" truck transporting logs of Eucalyptus sp. and Pinus sp. with 3.60 and 7.20 m lengths. The results showed that the time consumed by trucks waiting for loading and unloading operations affected the reduced productivity and increased transport costs. The length wood influence is variable that affected directly the loading and unloading operations.

Keywords: Productivity; cost; logistics; optimization.
\end{abstract}

\section{INTRODUÇÃO}

O transporte é o elemento de grande importância na composição do custo logístico, sendo o frete responsável por uma média de $60 \%$ do gasto logístico na maioria das empresas transportadoras (RODRIGUES, 2007). No Brasil, o transporte de cargas é exclusivamente dependente do modal rodoviário, sendo responsável por $61,1 \%$ da carga total transportada (CNT, 2015).

Tal fato deve-se à extensa malha rodoviária existente em nosso país, à oferta de uma grande variedade de veículos com diferentes capacidades de carga, à adaptabilidade dos veículos às estradas, o menor preço de instalação do modal rodoviário e à flexibilidade na escolha de rotas (SEIXAS, 1992; MACHADO et al., 2009). Entretanto, as condições insatisfatórias desse sistema têm onerado os produtos brasileiros com elevados custos de frete e de manutenção dos veículos, reduzindo a sua competitividade (ELLER et al., 2011).

No caso específico do setor florestal, o transporte principal consiste na movimentação da madeira na forma de toras, normalmente com diferentes espécies e tamanhos, das áreas produtoras (fazendas) até o local de consumo final (indústrias). Esse tipo de transporte é influenciado por diversos fatores, tais como distância de transporte, padrão de qualidade das estradas, tipos de veículo, carregamento e descarregamento e sortimentos da madeira, entre outros, que afetam o desempenho dos veículos e os custos do transporte (MACHADO et al., 2009; ALVES et al., 2013).

Silversides (1976) afirma que o principal fator que afeta o custo do transporte é a distância percorrida pelos veículos, sendo que quanto maior o trajeto, maior o custo unitário por volume de madeira transportada, podendo tal custo ser reduzido com o uso de veículos de maior potência e capacidade de carga. Machado et al. (2009) diz que os altos custos do transporte florestal rodoviário são ocasionados principalmente pela sua especificidade de carga e exclusividade de frete, pois, normalmente, os veículos são adaptados para o transporte

FLOREST A, Curitiba, PR, v. 46, n. 3, p. 297 - 305, jul. / set. 2016.

Lopes, E. da S. et al.

ISSN eletrônico 1982-4688

DOI: $10.5380 /$ rf.v46i3.36826 
somente de madeira e trafegam carregados somente em um único sentido, elevando, assim, os custos do transporte, em comparação com outros produtos. Já Seixas (1992) ressalta o fato de a madeira ser um produto de valor específico relativamente baixo quando comparado aos outros produtos, ou seja, o volume transportado é alto em relação ao baixo valor da carga, elevando, assim, o custo do transporte para as empresas transportadoras.

Machado et al. (2006), avaliando o desempenho operacional de veículos no transporte de madeira, relatou que os fatores de maior influência na produtividade foram o número de carregadores florestais disponíveis no campo para realização do carregamento e a qualidade das estradas florestais. Já Silva et al. (2007), estudando o raio econômico do transporte de madeira para diferentes tipos de veículos, concluíram que o rodotrem apresentou melhor desempenho, devido à sua maior capacidade de carga, com redução significativa dos custos, sendo indicado para o transporte em longas distâncias.

Machado e Lopes (2000), ao estudarem a influência do comprimento da madeira nos custos do transporte, afirmam que, quanto maior o comprimento das toras, menores são os custos do transporte, devido, principalmente, ao melhor aproveitamento da carga sobre os veículos. Alves et al. (2013), por sua vez, ao compararem o desempenho de um tritrem ao de um rodotrem de mesma capacidade de carga, transportando toras nos comprimentos de 6,0 e 2,2 m, respectivamente, concluíram que o tritrem apresentou os menores custos transportando toras de maiores comprimentos.

Portanto, diante da importância do transporte rodoviário florestal na composição do custo logístico, objetivou-se, neste trabalho, realizar uma avaliação técnica e de custos do transporte de madeira de diferentes espécies e sortimentos, gerando informações que possam contribuir com os gestores no aumento da eficiência operacional e da produtividade e na redução dos custos do transporte.

\section{MATERIAL E MÉTODOS}

\section{Área de estudo}

Este trabalho foi realizado em uma empresa transportadora responsável pelo abastecimento de uma indústria de celulose e papel da região dos Campos Gerais, Paraná, localizada nas coordenadas geográficas de latitude $24^{\circ} 19^{\prime} 37^{\prime \prime} \mathrm{S}$ e longitude $50^{\circ} 36^{\prime} 58^{\prime \prime} \mathrm{W}$ e com altitude média de $760 \mathrm{~m}$.

O clima predominante na região de estudo, de acordo com o sistema de classificação climática de Köppen, está situado entre a região Cfa e Cfb, apresentando temperatura média no mês mais frio de $18^{\circ} \mathrm{C}$ (mesotérmico) e temperatura média no mês mais quente de $22^{\circ} \mathrm{C}$, com precipitação média anual entre 1.400 e $1.600 \mathrm{~mm}$.

As estradas da empresa possuíam pistas simples, em que $70 \%$ dos trechos trafegados pelos veículos de transporte possuíam pavimentação com revestimento primário, caracterizados pelas estradas principais e alguns trechos de estradas secundárias, enquanto $30 \%$ eram não pavimentados, caracterizados pelas estradas terciárias e divisores de talhão.

\section{Caracterização do veículo}

Foi avaliado um veículo articulado denominado "bitrem", composto por um cavalo mecânico com potência no motor de $420 \mathrm{HP}$ e equipado com dois semirreboques de 19,80 m de comprimento, peso líquido da carga de 37 toneladas e peso bruto total combinado (PBTC) de 57 toneladas.

Foi estudado o transporte de toras de Eucalyptus sp. e Pinus sp., cuja madeira era destinada para a produção de celulose e papel. Os sortimentos da madeira com as respectivas dimensões e as distâncias médias de transporte são apresentados na tabela 1. As distâncias médias de transporte foram obtidas por meio da quilometragem rodada pelos veículos obtidas dos boletins diários de bordo, considerando as distâncias médias de viagem vazio e carregado (ida e volta).

Tabela 1. Sortimentos das toras e distância média de transporte.

Table 1. Assortments of logs and medium distance transport.

\begin{tabular}{lcccc}
\hline Espécie & Sortimento & Comprimento $(\mathbf{m})$ & Diâmetro $(\mathbf{c m})$ & Distância média transporte (km) \\
\hline \multirow{2}{*}{ Eucalyptus sp. } & $\mathrm{A}$ & 3,60 & 8 a 35 & 48,8 \\
& $\mathrm{~B}$ & 7,20 & 8 a 35 & 40,6 \\
\hline \multirow{2}{*}{ Pinus sp. } & $\mathrm{A}$ & 3,60 & 8 a 35 & 31,6 \\
& $\mathrm{~B}$ & 7,20 & 8 a 35 & 39,0 \\
\hline \multirow{2}{*}{ Média } & & & & 40,0 \\
\hline
\end{tabular}

\section{Procedimento amostral}

Inicialmente foi realizado um estudo piloto de tempos e movimentos do transporte rodoviário florestal pelo método de cronometragem de tempos contínuos, de modo a definir o número mínimo de ciclos operacionais necessários para um erro de amostragem máximo de 5\%, por meio da expressão proposta por Conaw (1977): 


$$
\mathrm{n} \geq \frac{\mathrm{t}^{2} \mathrm{xCV}}{\mathrm{E}^{2}}
$$

em que: $\mathrm{n}=$ número mínimo de ciclos de transporte; $\mathrm{t}=$ valor de $\mathrm{t}$, para o nível de probabilidade desejado e $(\mathrm{n}-$ 1) graus de liberdade; $\mathrm{CV}=$ coeficiente de variação (\%); e $\mathrm{E}=$ erro admissível (\%).

A partir do número mínimo de ciclos operacionais definidos pelo estudo piloto, foram selecionados de forma aleatória, no banco de dados da empresa, 267 e 320 ciclos operacionais referentes ao transporte de Eucalyptus sp. e 170 e 185 ciclos operacionais no transporte de Pinus sp., ambos nos sortimentos A e B, respectivamente. O banco de dados foi gerado a partir dos diários de bordo dos veículos preenchidos pelos próprios motoristas durante a realização das viagens ou ciclo operacional.

\section{Análise técnica}

A análise técnica do transporte rodoviário florestal baseou-se nos dados obtidos dos boletins diários dos veículos, sendo registrados os seguintes tempos parciais do ciclo operacional:

- Viagem vazio (VV): correspondeu ao tempo consumido pelos veículos durante o deslocamento da indústria até o local de carregamento localizado no campo.

- Carregamento (CR): correspondeu ao tempo consumido pelos veículos durante a operação de carregamento no campo, composto pelos tempos de espera na fila (ET) e o carregamento propriamente dito (CV).

- Viagem carregado (VC): correspondeu ao tempo consumido pelos veículos durante o deslocamento do ponto de carregamento no campo até a portaria da indústria.

- Descarregamento (DR): correspondeu ao tempo consumido pelos veículos durante a operação de descarregamento na indústria, composto pelos tempos parciais de esperas em filas para entrada no controle de pesagem (CB), entre a pesagem e o local de descarregamento (BD), descarregamento propriamente dito (DV) e a parada no ponto de apoio para o preenchimento de nova ficha para realização da próxima viagem (PA).

Foram ainda considerados os tempos improdutivos (IMP), que corresponderam às interrupções operacionais (abastecimento, aguardando nota fiscal, borracharia, troca de equipe, espera de carga, manutenção corretiva, manutenção preventiva etc.) e não operacionais (necessidades fisiológicas e pausas pessoais).

A análise do percentual dos tempos do ciclo operacional do transporte em função da espécie e sortimentos da madeira foi realizada para uma distância média de $40 \mathrm{~km}$ (total de $80 \mathrm{~km}$ contemplando as viagens de ida e volta), sendo então comparados os tempos das atividades operacionais.

De posse dos dados de tempos e das distâncias percorridas pelos veículos, foram obtidas as velocidades médias e determinadas a disponibilidade mecânica, a eficiência operacional e a produtividade dos veículos nas diferentes condições operacionais.

Velocidade média do transporte

A velocidade média dos veículos no transporte da madeira foi obtida a partir da distância percorrida durante as viagens vazio e carregado em relação ao tempo total consumido, por meio da expressão proposta por MACHADO et al. (2009):

$$
\mathrm{VM}=\frac{\mathrm{DM}}{\mathrm{TV}}
$$

em que: $\mathrm{VM}=$ velocidade média $\left(\mathrm{km} \cdot \mathrm{h}^{-1}\right) ; \mathrm{DM}=$ distância percorrida $(\mathrm{km}) ;$ e TV = tempo de viagem (horas).

Disponibilidade mecânica

A disponibilidade mecânica foi obtida pela percentagem de tempo de trabalho programado em que os veículos estavam mecanicamente disponíveis para o transporte, desconsiderando-se os tempos em que estiveram parados em manutenção preventiva e/ou corretiva, por meio da expressão adaptada de Santos et al. (2013):

$$
\mathrm{DM}=\frac{\mathrm{TP}-\mathrm{TM}}{\mathrm{TP}} \times 100
$$

em que: $\mathrm{DM}=$ disponibilidade mecânica $(\%) ; \mathrm{TP}=$ tempo programado para o trabalho (horas); e $\mathrm{TM}=$ tempo de manutenção preventiva e/ou corretiva (horas).

\section{Eficiência operacional}

A eficiência operacional foi determinada pela relação entre o tempo em que os veículos estiveram realizando o transporte de forma efetiva em relação ao tempo programado, ou seja, o tempo em que os veículos encontravam-se em operação, desconsiderando-se as interrupções operacionais e não operacionais, por meio da expressão adaptada de Santos et al. (2013):

FLORESTA, Curitiba, PR, v. 46, n. 3, p. 297 - 305, jul. / set. 2016.

Lopes, E. da S. et al.

ISSN eletrônico 1982-4688

DOI: $10.5380 /$ rf.v46i3.36826 


$$
\mathrm{EO}=\frac{\mathrm{TE}}{\mathrm{TP}} \times 100
$$

em que: $\mathrm{EO}=$ eficiência operacional $(\%) ; \mathrm{TE}=$ tempo de trabalho efetivo (horas); e $\mathrm{TP}=$ tempo programado (horas).

\section{Produtividade}

A produtividade foi determinada pela relação entre a quantidade de madeira transportada pelos veículos, em toneladas, em relação à quilometragem percorrida em cada ciclo operacional, sendo então corrigida pela eficiência operacional, por meio da expressão adaptado de Machado et al. (2009):

$$
\mathrm{P}=\frac{\mathrm{CC}}{\frac{\mathrm{DVv}}{\mathrm{VVv}}+\frac{\mathrm{DVc}}{\mathrm{VVc}}+\mathrm{Tc}+\mathrm{Td}} \times \mathrm{EO}
$$

em que: $\mathrm{P}=$ produtividade do veículo $\left(\mathrm{t} \cdot \mathrm{h}^{-1}\right) ; \mathrm{CC}=$ quantidade de carga transportada $(\mathrm{t}) ; \mathrm{DVv}=$ distância percorrida pelo veículo vazio $(\mathrm{km}) ; \mathrm{VVv}=$ velocidade média do veículo na viagem vazio $\left(\mathrm{km} \cdot \mathrm{h}^{-1}\right)$; $\mathrm{DV} c=$ distância percorrida pelo veículo carregado $(\mathrm{km}) ; \mathrm{VVc}=$ velocidade média do veículo na viagem carregado $\left(\mathrm{km} \cdot \mathrm{h}^{-1}\right) ; \mathrm{Tc}=$ tempo de carregamento $(\mathrm{h}) ; \mathrm{Td}=$ tempo de descarregamento $(\mathrm{h}) ;$ e EO = eficiência operacional.

\section{Análise de custos}

A análise de custos foi realizada pelo método FAO/Norte Americano adaptado por Braga et. al. (2011) para bitrem modelo $420 \mathrm{HP}$. Para obtenção dos custos operacionais, foram considerados os custos fixos (depreciação, juros, seguros, impostos e mão de obra), os custos variáveis (combustível, graxas e lubrificantes, manutenção e consertos e pneus) e os custos de administração.

A determinação do custo de espera (CE) foi feita pela soma dos custos de juros, seguros, taxa e impostos, depreciação e mão de obra, enquanto o custo de viagem (CV) foi determinado pelo somatório do custo operacional com os demais componentes dos custos.

O custo de transporte foi obtido pela razão da carga transportada por meio da seguinte expressão proposta por Machado et al. (2009):

$$
\mathrm{Ct}=\frac{(\mathrm{TE} \cdot \mathrm{CE})+(\mathrm{TV} \cdot \mathrm{CV})}{60 \cdot \mathrm{Vc}}
$$

em que: $\mathrm{Ct}=$ custo de transporte $\left(\mathrm{R} \$ \cdot \mathrm{t}^{-1}\right) ; \mathrm{TE}=$ tempo de espera (he); $\mathrm{CE}=$ custo de espera $\left(\mathrm{R} \$ . h \mathrm{e}^{-1}\right)$; $\mathrm{TV}=$ tempo de viagem (he); $\mathrm{CV}=$ custo de viagem $\left(\mathrm{R} \$ \cdot \mathrm{he}^{-1}\right) ; \mathrm{e} \mathrm{Vc}=$ capacidade de carga do veículo $(\mathrm{t})$.

Em seguida, foi obtido o custo de transporte por quilômetro rodado, que indica o custo de cada tonelada transportada por quilômetro rodado, sendo obtido por meio da expressão proposta por Machado et al. (2009):

$$
\mathrm{CTK}=\frac{\mathrm{Ct}}{\mathrm{D}}
$$

em que: $\mathrm{CTK}=$ custo de transporte por tonelada e quilômetro rodado $\left(\mathrm{R} \$ \cdot \mathrm{t}^{-1} \cdot \mathrm{km}^{-1}\right) ; \mathrm{Ct}=$ custo de transporte por tonelada $\left(\mathrm{R} \$ . \mathrm{t}^{-1}\right)$; e $\mathrm{D}=$ distância percorrida pelos veículos $(\mathrm{km})$.

\section{Análise estatística}

Foi aplicado o teste estatístico de Bartlett $(\alpha=5 \%)$ e na análise da distribuição dos dados utilizou-se o teste de normalidade de Kolmogorov-Smirnov $(\alpha=5 \%)$, mediante o software ASSISTAT. Em seguida, para comparação dos dados entre as espécies e sortimentos de madeira, foi utilizado o teste de Wilcoxon-MannWhitney, que é um teste não paramétrico alternativo ao teste t-Student para comparação das médias de duas amostras independentes.

\section{RESULTADOS}

Os tempos percentuais do ciclo operacional do transporte de Eucalyptus e Pinus nos diferentes sortimentos, considerando uma distância total média de $80 \mathrm{~km}$ (ida + volta) são apresentados na tabela 2. Como pode ser observado, houve um equilíbrio entre os tempos de viagem vazio e carregado em relação às operações de carregamento e descarregamento. No transporte das toras de Eucalyptus, independentemente dos sortimentos 
transportados, os tempos de viagem vazio e carregado representaram, em média, 23,9 e 29,3\% do tempo total do ciclo operacional de transporte, enquanto no Pinus ocuparam 23,6 e 29,6\%, respectivamente, não havendo diferença significativa nos tempos entre espécies e sortimentos.

Tabela 2. Distribuição percentual média dos tempos do ciclo operacional de transporte para as diferentes espécies e sortimentos de madeira.

Table 2. Average percentage distribution of the transportation operational cycle for different species and wood assortments.

\begin{tabular}{|c|c|c|c|c|c|c|c|}
\hline Espécie & Sortimento da madeira & $\begin{array}{l}V V \\
(\%)\end{array}$ & $\begin{array}{l}\text { CR } \\
(\%)\end{array}$ & $\begin{array}{l}\mathrm{VC} \\
(\%)\end{array}$ & $\begin{array}{l}\text { DR } \\
(\%)\end{array}$ & $\begin{array}{l}\text { INT } \\
(\%)\end{array}$ & TOTAL \\
\hline \multirow{3}{*}{ Eucalyptus } & $\mathrm{A}(3,60)$ & 23,2 & 17,6 & 28,6 & 22,9 & 7,7 & 100,0 \\
\hline & $\mathrm{B}(7,20)$ & 23,9 & 16,5 & 30,0 & 20,5 & 9,1 & 100,0 \\
\hline & Média & 23,6 & 17,1 & 29,3 & 21,7 & 8,4 & 100,0 \\
\hline \multirow{2}{*}{ Pinus } & $\mathrm{A}(3,60)$ & 25,2 & 19,2 & 27,3 & 20,8 & 7,5 & 100,0 \\
\hline & $\mathrm{B}(7,20)$ & 21,9 & 18,6 & 31,8 & 17,8 & 9,9 & 100,0 \\
\hline Média & & 23,6 & 18,9 & 29,6 & 19,3 & 8,7 & 100,0 \\
\hline \multirow{3}{*}{ P-valor } & $\begin{array}{l}\text { Entre sortimentos } \\
\text { (Eucalyptus) }\end{array}$ & $0,79589^{\mathrm{ns}}$ & $0,01967^{*}$ & $0,16783^{\text {ns }}$ & $0,00815^{*}$ & $0,51442^{\mathrm{ns}}$ & $0,22204^{\mathrm{ns}}$ \\
\hline & $\begin{array}{l}\text { Entre sortimentos } \\
\text { (Pinus) }\end{array}$ & $0,13957^{\mathrm{ns}}$ & $0,0006^{*}$ & $0,10321^{\mathrm{ns}}$ & $0,00092 *$ & $0,14752^{\mathrm{ns}}$ & $0,19248^{\mathrm{ns}}$ \\
\hline & Entre espécies & $0,27353^{\text {ns }}$ & $0,10778^{\mathrm{ns}}$ & $0,0619^{\mathrm{ns}}$ & $0,22155^{\mathrm{ns}}$ & $0,51188^{\mathrm{ns}}$ & $0,10314^{\mathrm{ns}}$ \\
\hline
\end{tabular}

${ }^{\text {ns }}$ não significativo; $*$ significativo a $5 \%$. VV = viagem vazio. $\mathrm{CR}=$ carregamento; $\mathrm{VC}=$ viagem carregado; $\mathrm{DR}=$ descarregamento; INT $=$ interrupções.

Os tempos médios do ciclo operacional dos veículos no transporte das toras de Eucalyptus foram de 4,73 e 5,65 h nos sortimentos A e B, respectivamente, enquanto no Pinus foram de 4,83 e 5,28 horas, não havendo também diferença significativa entre as espécies e os sortimentos estudados.

Em relação à etapa de carregamento da madeira especificamente (Figura 1), é possível notar o elevado tempo em que os veículos permaneceram nas filas de espera, que pode ser explicado pelo elevado número de veículos de transporte em relação ao baixo número de carregadores disponíveis no campo.
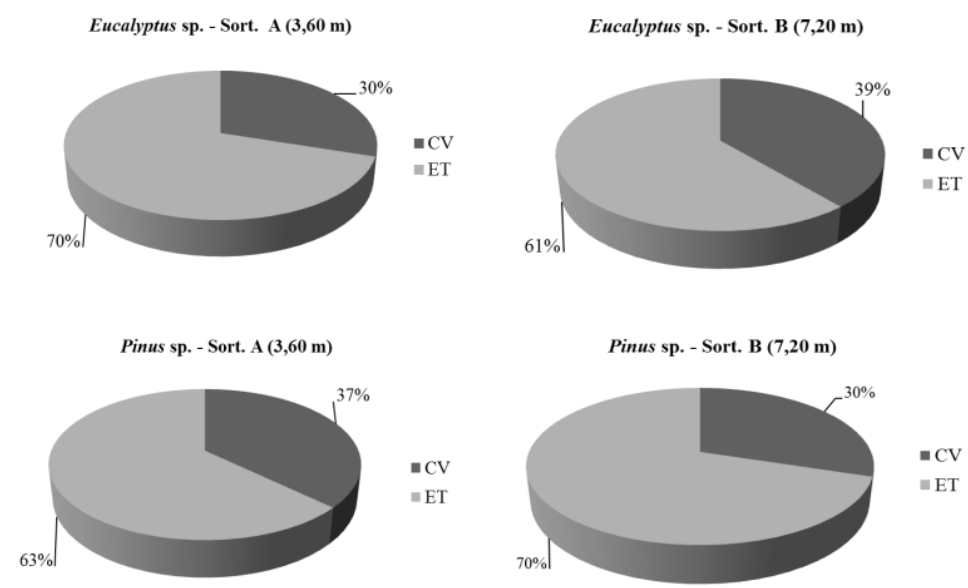

$\mathrm{CV}=$ tempo de carregamento propriamente dito; $\mathrm{ET}=$ tempo na fila de espera

Figura 1. Distribuição percentual do tempo consumido pelos veículos de transporte na operação de carregamento de diferentes espécies e sortimentos de madeira.

Figure 1. Percentage distribution of time consumed by truck transportation in loading operation for different species and wood assortments.

Em relação à operação de descarregamento (Figura 2), verifica-se que o tempo consumido pelos veículos nas filas de espera desde a entrada do ponto de controle até a pesagem (balança), e desta em relação ao local de descarregamento propriamente dito, ocupou mais da metade do tempo total de permanência dos veículos na indústria, caracterizando, portanto, um problema operacional que afeta a produtividade dos veículos, causando elevação nos custos do transporte de madeira. 

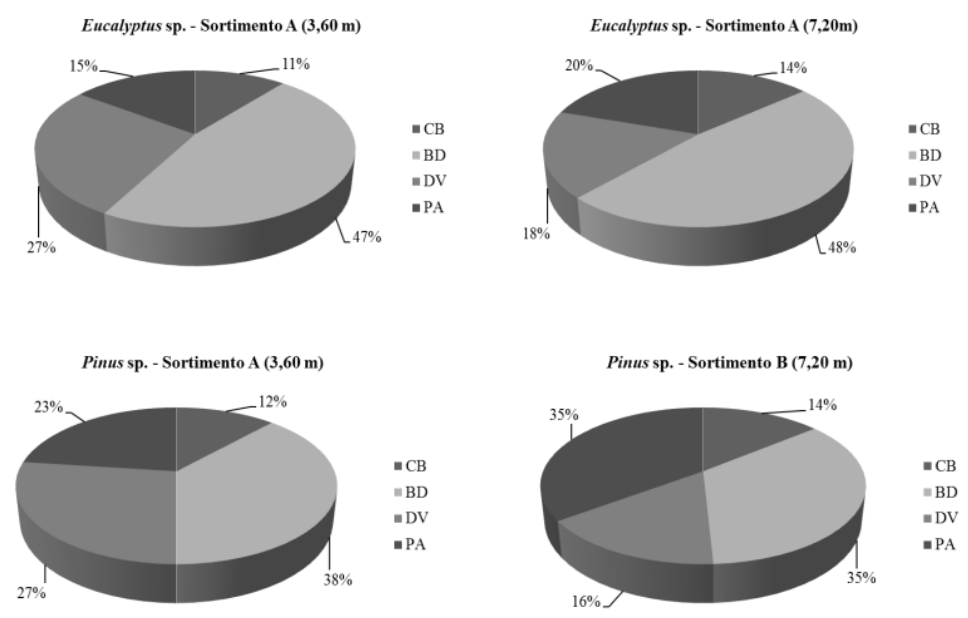

$\mathrm{CB}=$ tempo de espera em fila da entrada do controle até a pesagem; $\mathrm{BD}=$ tempo de espera fila da saída da pesagem até o local do descarregamento; $\mathrm{DV}=$ tempo de descarregamento propriamente dito; $\mathrm{PA}=$ tempo de parada no ponto de apoio.

Figura 2. Distribuição percentual do tempo consumido pelos veículos de transporte na operação de descarregamento de diferentes espécies e sortimentos de madeira.

Figure 2. Percentage distribution of time consumed by truck transportation in unloading operation for different species and wood assortments.

Ao se analisar o efeito dos diferentes comprimentos da madeira nas operações de carregamento e descarregamento, nota-se que, nas toras de Eucalyptus de menor comprimento (3,60 m), os tempos médios de carregamento e descarregamento foram de 23,8 e 23,9 minutos, enquanto nas toras de maior comprimento $(7,20$ m) foram de 35,3 e 12,9 minutos. Já nas toras de Pinus de menor comprimento, os tempos de carregamento e descarregamento foram de 31,4 e 19,4 minutos, enquanto nas de maior comprimento foram de 16,4 e 9,4 minutos, respectivamente.

Portanto, diante desses resultados, é possível verificar que, de modo geral, os tempos de descarregamento foram inferiores aos de carregamento. Além disso, percebe-se que o aumento do comprimento da madeira contribuiu para a redução dos tempos de carregamento e descarregamento, exceto no carregamento de Eucalyptus de 7,20 m, em que o maior tempo pode ser atribuído ao nível de experiência do operador (Tabela 3).

Tabela 3. Tempos médios de carregamento e descarregamento de Pinus e Eucalyptus nos sortimentos avaliados. Table 3. Average time of loading and unloading of Pinus and Eucalyptus in the assortments evaluated.

\begin{tabular}{lrrrrrr}
\hline Comparação & \multicolumn{2}{l}{$\begin{array}{l}\text { Média do tempo de } \\
\text { carregamento (min.) }\end{array}$} & \multicolumn{2}{l}{$\begin{array}{l}\text { Média do tempo de } \\
\text { descarregamento (min.) }\end{array}$} & \multicolumn{2}{l}{ P-valor } \\
\hline Entre sortimentos & $3,60 \mathrm{~m}$ & $7,20 \mathrm{~m}$ & $3,60 \mathrm{~m}$ & $7,20 \mathrm{~m}$ & Carregamento & Descarregamento \\
\hline Eucalyptus & 23,8 & 35,3 & 23,9 & 12,9 & $0,01967^{*}$ & $0,00815^{*}$ \\
Pinus & 31,4 & 16,4 & 19,4 & 9,4 & $0,00060^{*}$ & $0,00092^{*}$ \\
\hline Entre espécies & Eucalyptus & Pinus & Eucalyptus & Pinus & Carregamento & Descarregamento \\
\hline Sortimentos agrupados & 29,5 & 23,9 & 18,4 & 14,4 & $0,10778 \mathrm{~ns}$ & $0,22155 \mathrm{~ns}$ \\
\hline
\end{tabular}

${ }^{\mathrm{ns}}$ não significativo; * significativo a $5 \%$.

Na tabela 4 é possível notar que, independentemente da espécie e sortimento da madeira, as interrupções no transporte ocorreram de forma mais significativa durante a permanência dos veículos nas filas de espera para 0 controle, além do elevado tempo consumido pelos veículos com as manutenções corretivas e preventivas.

A velocidade média dos veículos nas viagens vazio e carregado, a distância média percorrida, a disponibilidade mecânica, a eficiência operacional e o custo do transporte nas diferentes condições estudadas são apresentados na tabela 5. É possível observar uma maior velocidade dos veículos durante a viagem vazio, com diferença média de $35 \%$ em relação à viagem carregado, o que mostra, portanto, a influência da carga transportada no desempenho dos veículos. 
Tabela 4. Distribuição percentual das interrupções operacionais e não operacionais do transporte de madeira nas diferentes espécies e sortimentos.

Table 4. Percentage distribution of operational and non-operational interruptions of wood transportation in the different species and assortments.

\begin{tabular}{lcccc}
\hline \multirow{2}{*}{ Elemento } & \multicolumn{3}{c}{ Pucalyptus sp. } & \multicolumn{3}{c}{ sp. } \\
\cline { 2 - 5 } & Sortimento A (\%) & Sortimento B (\%) & Sortimento A (\%) & Sortimento B (\%) \\
\hline Abastecimento & 0,6 & 1,4 & 1,3 & 0,7 \\
Aguardando NF & 8,2 & 2,1 & 10,4 & 4,5 \\
Borracharia & 11,0 & 10,1 & 4,3 & 8,3 \\
Troca de equipe & 11,4 & 11,5 & 5,3 & 3,3 \\
Espera de carga no controle & 28,2 & 44,6 & 40,7 & 37,8 \\
Manutenção corretiva & 19,2 & 13,2 & 18,7 & 20,6 \\
Manutenção preventiva & 16,1 & 15,2 & 17,8 & 20,9 \\
Outros & 5,4 & 1,9 & 2,1 & 3,8 \\
\hline
\end{tabular}

A velocidade média do veículo vazio e carregado foi de $43,5 \mathrm{~km} \cdot \mathrm{h}^{-1}$ e $27,0 \mathrm{~km} \cdot \mathrm{h}^{-1}$ para Eucalyptus sp., e de $31,2 \mathrm{~km} . \mathrm{h}^{-1}$ e $21,8 \mathrm{~km} \cdot \mathrm{h}^{-1}$ para Pinus sp., respectivamente. Nota-se que essa velocidade influenciou o custo de transporte, ou seja, o Pinus apresentou uma velocidade média menor e, consequentemente, um maior custo de transporte $\left(\mathrm{R} \$ 15,33 \cdot \mathrm{t}^{-1}\right)$.

Tabela 5. Velocidade média dos veículos vazio e carregado, disponibilidade mecânica e eficiência operacional e custos do transporte de madeira.

Table 5. Average speed of empty and loaded vehicles, mechanical availability, operational efficiency and cost of wood transportation.

\begin{tabular}{|c|c|c|c|c|c|c|c|c|}
\hline Espécie & Sortimento & $\begin{array}{c}\text { VM vazio } \\
\left(\mathbf{k m} \cdot \mathbf{h}^{-1}\right)\end{array}$ & $\begin{array}{c}\mathrm{VM} \\
\text { carregado } \\
\left(\mathbf{k m} \cdot \mathrm{h}^{-1}\right) \\
\end{array}$ & $\begin{array}{c}\text { Distância } \\
\text { média } \\
(\mathbf{k m})\end{array}$ & $\begin{array}{l}\text { DM } \\
(\%)\end{array}$ & $\begin{array}{l}\text { EO } \\
(\%)\end{array}$ & $\begin{array}{c}\mathbf{C T} \\
\left(\mathbf{R} \$ . \mathbf{t}^{-1}\right)\end{array}$ & $\begin{array}{c}\text { CTK } \\
\left(\mathrm{R} \$ \cdot \mathrm{t}^{-1} \cdot \mathrm{km}^{-1}\right)\end{array}$ \\
\hline \multirow{3}{*}{ Eucalyptus sp. } & $\mathrm{A}(3,60 \mathrm{~m})$ & 44,4 & 27,9 & 48,8 & 97,1 & 92,4 & 14,72 & 0,151 \\
\hline & $\mathrm{B}(7,20 \mathrm{~m})$ & 42,5 & 26,0 & 40,6 & 97,4 & 91,0 & 11,03 & 0,136 \\
\hline & Média & 43,5 & 27,0 & 44,7 & 97,3 & 91,7 & 12,90 & 0,144 \\
\hline \multirow{3}{*}{ Pinus sp. } & $\mathrm{A}(3,60 \mathrm{~m})$ & 31,6 & 22,3 & 31,6 & 97,2 & 92,7 & 13,28 & 0,210 \\
\hline & $\mathrm{B}(7,20 \mathrm{~m})$ & 30,7 & 21,3 & 39,0 & 95,4 & 90,1 & 15,33 & 0,149 \\
\hline & Média & 31,2 & 21,8 & 35,3 & 96,3 & 91,4 & 14,30 & 0,179 \\
\hline Média geral & & 37,3 & 24,4 & 40,0 & 96,8 & 91,6 & 13,59 & 0,162 \\
\hline
\end{tabular}

$\overline{\mathrm{VM}}=$ velocidade média; $\mathrm{DM}=$ disponibilidade mecânica; $\mathrm{EO}=$ eficiência operacional; $\mathrm{CT}=$ custo de transporte; CTK = custo de transporte por tonelada/quilômetro.

A disponibilidade mecânica média dos veículos foi de $96,8 \%$ para ambas as espécies e sortimentos estudados, sendo considerados elevados, apesar da ocorrência de paradas para manutenção preventiva e corretiva. Isso pode ser explicado pelo fato de os veículos utilizados serem novos, enquanto a eficiência operacional média foi de $91,6 \%$, estando acima do mínimo exigido pela empresa contratante $(85 \%)$. Tais valores também se encontram acima dos resultados obtidos por Alves et al. (2013), que obtiveram uma eficiência operacional que variou de 88,5 a 90,4\% para um "bitrem" transportando toras de Eucalyptus no estado de Minas Gerais.

A produtividade média dos veículos nas condições estudadas foi afetada pela distância percorrida (Figura 3). Como pode ser visto, independentemente da espécie e sortimento da madeira, o aumento da distância de transporte ocasionou redução na produtividade, explicada pelo maior tempo de duração do ciclo operacional e diminuição do número de viagens. Por outro lado, percebe-se que o aumento do comprimento da madeira contribuiu para a melhoria da produtividade dos veículos, sendo mais evidente no transporte de Pinus, enquanto as operações de carregamento e descarregamento afetou diretamente a produtividade dos veículos.

O custo operacional médio dos veículos no transporte das toras de Eucalyptus sp. foi de R $\$ 12,90 . \mathrm{t}^{-1}$, perfazendo um custo médio por quilômetro rodado de $\mathrm{R} \$ 0,14 \cdot \mathrm{t}^{-1} \cdot \mathrm{km}^{-1}$, enquanto no transporte de Pinus os custos médios foram de $\mathrm{R} \$ 14,30 . \mathrm{t}^{-1}$ e de $\mathrm{R} \$ 0,18 \cdot \mathrm{t}^{-1} \cdot \mathrm{km}^{-1}$. Tais resultados podem ser atribuídos ao melhor desempenho dos veículos no transporte das toras de Eucalyptus sp., que, mesmo numa maior distância percorrida $(44,7 \mathrm{~km})$, desenvolveram maior velocidade, contribuindo para a redução do tempo médio de viagem.

O custo médio de transporte em função do comprimento das toras transportadas, independentemente da espécie, foi de $R \$ 14,00 \cdot \mathrm{t}^{-1}$ ou $\mathrm{R} \$ 0,18 \cdot \mathrm{t}^{-1} \cdot \mathrm{km}^{-1}$ e de $\mathrm{R} \$ 13,18 \cdot \mathrm{t}^{-1}$ e $\mathrm{R} \$ 0,14 \cdot \mathrm{t}^{-1} \cdot \mathrm{km}^{-1}$ nos sortimentos $\mathrm{A}$ e $\mathrm{B}$, respectivamente. É importante ressaltar que, no transporte de ambos os tipos de madeira, os veículos 
desenvolveram uma velocidade média semelhante, sendo que a diferença no custo deveu-se, principalmente, aos tempos de carregamento e descarregamento, que são diretamente influenciados pelo comprimento da madeira.

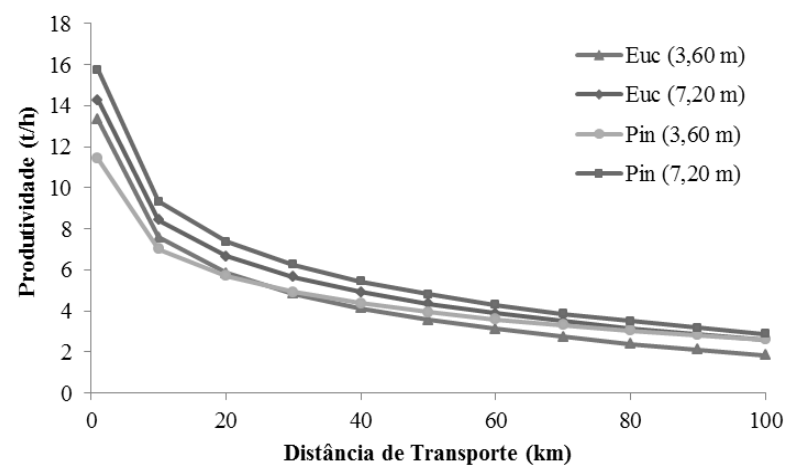

Figura 3. Produtividade média dos veículos no transporte de toras nas diferentes distâncias de transporte e sortimentos de madeira, para Eucalyptus sp. e Pinus sp.

Figure 3. Average productivity of trucks in the transportation at different transport distances and wood assortments, for Eucalyptus sp. and Pinus sp.

\section{DISCUSSÃO}

Os veículos de transporte consumiram um elevado tempo para a realização das operações de carregamento e descarregamento em comparação com o tempo total do ciclo operacional, podendo tal resultado ser atribuído à menor distância de transporte $(40 \mathrm{~km})$. Nessas condições, Machado et al. (2009) diz que é normal que os veículos permaneçam, proporcionalmente, um maior tempo realizando as operações de carregamento e descarregamento, situação contrária que ocorre em longas distâncias, quando o uso de veículos de maior capacidade de carga torna-se mais vantajoso do ponto de vista econômico.

O elevado tempo consumido pelos veículos durante a operação de carregamento mostrou a necessidade de melhorias no planejamento logístico, por meio de uma melhor distribuição da frota, evitando que vários veículos cheguem ao mesmo tempo no campo para realização do carregamento, bem como da necessidade de aumento do número de carregadores florestais. Tal afirmação está de acordo com Machado et al. (2006), que diz que a formação de filas de espera não está somente relacionada ao número de veículos de transporte, mas também ao número de carregadores florestais disponíveis no campo e às condições das estradas, fatores que afetam diretamente o desempenho dos veículos de transporte rodoviário florestal.

Além disso, os resultados comprovaram a influência do comprimento da madeira nos tempos consumidos pelos veículos de transporte, principalmente na produtividade do carregamento e descarregamento, sendo um parâmetro importante na busca por melhorias no planejamento das operações para aumento da produtividade e redução dos custos do transporte. Tal resultado está em concordância com Machado e Lopes (2000), que ressaltam a influência do comprimento da madeira na produtividade e no custo das operações de transporte florestal.

O tempo dos veículos nas filas de espera nas operações de carregamento e descarregamento comprometeu a produtividade do transporte, sendo um indicativo da necessidade de melhorias no planejamento e no controle logístico, envolvendo as atividades de carregamento, transporte principal e descarregamento na indústria. Uma solução para que seja alcançada maior eficiência e redução dos tempos de espera em filas é o uso das tecnologias de comunicação, podendo, segundo Totti (2014), ser usados sistemas de comunicação operados pela internet, aparelhos de satélite e de comunicação móvel (GPRS), que permitem a obtenção de informações confiáveis e com maior rapidez, além de auxiliarem os gestores nas tomadas de decisões e controle das operações com maior eficiência e grau de acerto.

\section{CONCLUSÕES}

Com base nos resultados desta pesquisa, chegou-se às seguintes conclusões:

- A distância de transporte e o tempo de permanência dos veículos nas filas de espera contribuíram para o elevado tempo de carregamento e descarregamento da madeira, comprometendo a produtividade e os custos de transporte. 
- O baixo número de carregadores florestais disponíveis no campo em relação ao número de veículos de transporte em operação e a inadequada distribuição da frota contribuíram para o elevado tempo de permanência dos veículos em filas de espera, indicando a necessidade de melhorias no planejamento logístico.

- A velocidade média do veículo influenciou a produtividade e o custo, sendo mais evidente no transporte de eucalipto realizado em uma maior distância.

- O comprimento da madeira influenciou os tempos e a produtividade do carregamento e descarregamento, mostrando a necessidade de melhorias no planejamento do transporte florestal rodoviário realizado em curtas distâncias.

\section{REFERÊNCIAS}

ALVES, R. T.; FIEDLER, N. C.; SILVA, E. N.; LOPES, E. S.; CARMO, F. C. A. Análise técnica e de custos do transporte de madeira com diferentes composições veiculares. Revista Árvore, Viçosa, v. 37, n. 5, p. 897-904, 2013.

BRAGA, A. X. V.; SOUZA, M. A. de; BRAGA, D. P. G. Custo operacional de caminhão bitrem: aplicação atualizada e adaptada do método FAO/América do Norte. Custo@gronegócio on line, v. 7, n. 3, 2011.

CONAW, P.L. Estatística. São Paulo: Edgard Blucher, 1977. 264 p.

CONFEDERAÇÃO NACIONAL DO TRANSPORTE. Boletim Estatístico - CNT - Julho 2015. CNT: SEST: SENAT. Brasília, DF. 2013. Disponível em: <http://http://www.cnt.org.br/. Acesso em: 10/08/2015.

ELLER, R. A. G.; SOUSA JUNIOR, W. C.; CURI, M. L. C. Custos do transporte de carga no Brasil: rodoviário versus ferroviário. Revista de Literatura dos Transportes, v. 5, n. 1, p. 50-64, 2011.

MACHADO, C. C.; LOPES, E. S. Análise da influência do comprimento de toras de eucalipto na produtividade e custo da colheita e transporte florestal. Cerne, Lavras, v. 6, n. 2, p. 124-129, 2000.

MACHADO, C. C.; LOPES, E. S.; BIRRO, M. H. Elementos básicos do transporte florestal rodoviário. Viçosa: Editor UFV, 2. ed., 2009. 167 p.

MACHADO, R. R.; SILVA, M. L.; MACHADO, C. C.; LEITE, H. G. Avaliação do desempenho logístico do transporte rodoviário de madeira utilizando rede de petri em uma empresa florestal de Minas Gerais. Revista Árvore, Viçosa, v. 30, n. 6, p. 999-1008, 2006.

RODRIGUES, P. R. Introdução aos sistemas de transporte no Brasil e à logística internacional. São Paulo: Aduaneiras, 4. ed., 2007. 243 p.

SANTOS, P. H. A.; SOUZA, A. P.; MARZANO, F. L. C.; MINETTE, L. J. Produtividade e custos de extração de madeira de eucalipto. Revista Árvore, Viçosa, v. 37, n. 3, p. 511-518, 2013.

SEIXAS, F. Uma metodologia de seleção e dimensionamento da frota de veículos rodoviários para o transporte principal de madeira. $106 \mathrm{f}$. Tese (Doutorado em Engenharia de Transportes) - Universidade de São Paulo, São Carlos, 1992.

SILVA, M. L.; OLIVEIRA, R. J.; VALVERDE, S. R.; MACHADO, C. C.; PIRES, V. A. V. Análise do custo e do raio econômico de transporte de madeira de reflorestamentos para diferentes tipos de veículos. Revista Árvore, Viçosa, MG, v. 31, n. 6, p. 1073-1079, 2007.

SILVERSIDES, C. R. Survey of truks for raw material transport. World Wood, v. 17, n. 12, p. 42-52, 1976.

TOTTI, J. A. Sistema de controle logístico de frotas de caminhões florestais. In: SEMINÁRIO DE ATUALIZAÇÃO DE SISTEMAS DE COLHEITA DE MADEIRA E TRANSPORTE FLORESTAL, 17. Campinas. Anais... Campinas, 2014. 374 p. 